\title{
Microwave Depolarization above Sunspots
}

\author{
Jeongwoo Lee ${ }^{1}$ and Stephen M. White ${ }^{2}$ \\ ${ }^{1}$ Physics Department, New Jersey Institute of Technology, \\ Newark, NJ 07102, U.S.A. \\ email: leej@njit.edu \\ ${ }^{2}$ AFRL, Space Vehicles Directorate, Kirtland AFB, \\ Albuquerque, NM 87117, U.S.A. \\ email: afrl.rvb.pa@hanscom.af.mil
}

\begin{abstract}
Microwave emissions from sunspots are circularly polarized in the sense of rotation (right or left) determined by the polarity (north or south) of coronal magnetic fields. However, they may convert into unpolarized emissions under certain conditions of magnetic field and electron density in the corona, and this phenomenon of depolarization could be used to derive those parameters. We propose another diagnostic use of microwave depolarization based on the fact that an observed depolarization strip actually represents the coronal magnetic polarity inversion line (PIL) at the heights of effective mode coupling, and its location itself carries information on the distribution of magnetic polarity in the corona. To demonstrate this diagnostic utility we generate a set of magnetic field models for a complex active region with the observed line-of-sight magnetic fields but varying current density distribution and compare them with the $4.9 \mathrm{GHz}$ polarization map obtained with the Very Large Array (VLA). The field extrapolation predicts very different locations of the depolarization strip in the corona depending on the amount of electric currents assumed to exist in the photosphere. Such high sensitivity of microwave depolarization to the coronal magnetic field can therefore be useful for validating electric current density maps inferred from vector magnetic fields observed in the photosphere.
\end{abstract}

Keywords. Sun: corona, Sun: magnetic fields, Sun: radio radiation, polarization

\section{Introduction}

Magnetic fields in the sun and stars are usually measured from polarized spectral lines typically formed in the photosphere and the chromosphere (e.g. Zirin 1988, Dalgarno \& Layzer 1987). At greater coronal heights $\left(\sim 10^{5} \mathrm{~km}\right)$, however, the plasma is dilute and fully ionized, and fewer lines sensitive to magnetic field are available (Lin et al. 2000). Instead strong radiations exist at radio wavelengths and it is desirable to make use of their polarization to measure the magnetic field at those heights (Dulk \& McLean 1978). The coronal radio emissions appear in two natural modes: $\mathrm{X}$ mode which is polarized in the same sense that the electron gyrates about the magnetic field and $\mathrm{O}$ mode, polarized in the opposite sense. They are circularly polarized everywhere except for a narrow range of propagation angles orthogonal to the local magnetic field, and thus observed as either right or left-hand circular polarized (RCP and LCP hereafter) depending on the orientation of ambient magnetic field (Ratcliffe 1959). The X and O modes generally propagate independently of one other, and hence the polarization is unchanged during propagation. However, coupling between the two modes may occur when they pass through the region of magnetic fields orthogonal to the line of sight, called quasi-transverse (QT) layer (Kakinuma \& Swarup 1962). One particular outcome of the mode coupling is the complete depolarization of the emission which depends on the frequency, magnetic field, and density (Cohen, 1960). Many studies of mode coupling have thus far been focused on the use of specific relationship between density and magnetic field required for depolarization 

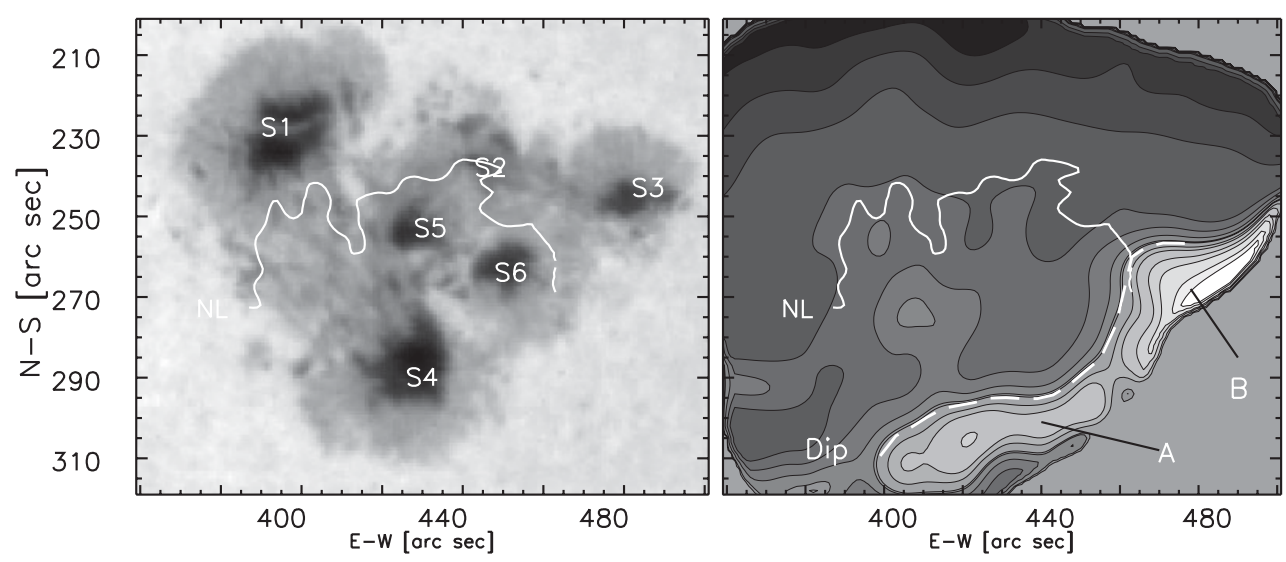

Figure 1. A white-light picture of a complex active region, AR 6615, obtained at the BBSO and a map of the degree of circular polarization taken with the VLA at $4.9 \mathrm{GHz}$. The depolarization lines, $V_{\mathrm{obs}}=0$, (dashed line) is noticeably shifted from the PIL of longitudinal photospheric magnetic fields (solid line). Thus, in this case, the observed sense of circular polarization does not well correspond to the magnetic polarity in the photosphere. The $(x, y)$ coordinates are geocentric coordinates in the west and the south, respectively, and $x$ and $y$ increase toward the west and the south, respectively. (from Lee et al. 1998)

in deriving one of these quantities (e.g., Kundu and Alissandrakis 1984, Alissandrakis \& Chiuderi Drago 1994, Alissandrakis et al. 1996, Ryabov et al. 1999). In this paper, we instead focus on the location of the depolarization strip as an important constraint on models for the large scale coronal magnetic field structure.

\section{Depolarization of Microwave Emissions}

Figure 1 shows the optical continuum image and a polarization map obtained for a complex active region AR 6615 from the Big Bear Solar Observatory (BBSO) and the Very Large Array (VLA), respectively. The radio polarization map was created from the conventional CLEAN images at $4.9 \mathrm{GHz}$ in which the white (black) regions are dominated by RCP (LCP). According to the longitudinal magnetogram (Fig. 2), the three northernmost spots (labeled S1-S3) are of negative (going into the Sun) polarity while the other three spots (S4-S6) are of positive (going out of the Sun) polarity and the bipolarity in this active region is generally north-south. Nonetheless, the negative polarization prevails over most of the active region so that the radio map is dominated by one polarity although the photospheric fields are bipolar. This phenomenon is expected to occur when the inversion line shifts so far to one side (southward in the present case) that the QT surface covers the southern half of the active region. In that case, waves emitted from the upgoing magnetic fields there start with RCP and then reverse their polarization to LCP on passing through the overlying QT surface. The waves emitted from the northern half of the active region start with LCP and never encounter any QT surface to remain as LCP all the way. Consequently most of the active region appears to be LCP. As a result, the only two emission regions which are not LCP are seen in the south end (region A) and on the western side (region B). The $V_{\text {obs }}=0$ line seen in region $\mathrm{A}$ is a long way from the photospheric PIL and the QT surface should be at a considerable height, where the density and magnetic field strength could be low enough to allow strong coupling to occur. We can thus conclude that the $V_{\mathrm{obs}}=0$ line in region $\mathrm{A}$ is due to depolarization. However, the corresponding line in region $\mathrm{B}$ cannot be understood 


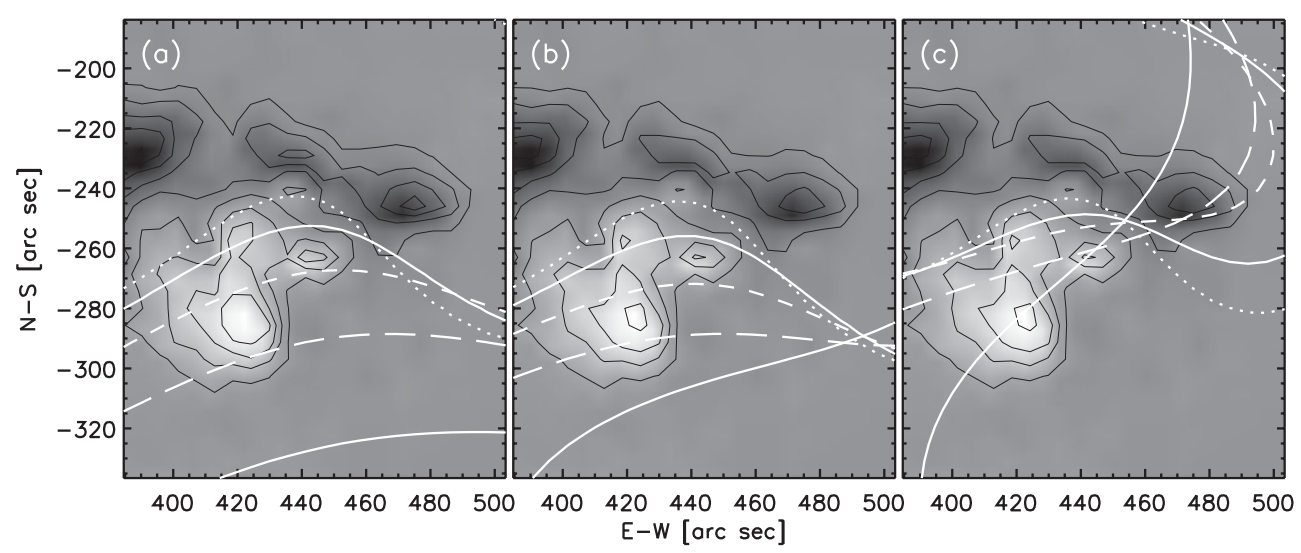

Figure 2. The coronal magnetic PILs predicted by the three magnetic field models (see text for description) overlaid on the photospheric magnetogram. The PILs correspond to heights of $h=30^{\prime \prime}$ (dotted lines), 60", 100", $140^{\prime \prime}$, and $180^{\prime \prime}$ (solid lines).

in the same way. This location is rather close to the photospheric PIL, and the waves emitted in region B would encounter the QT surface at a much lower height where the mode coupling is likely to be weak. Therefore the strong RCP seen in the western part of this active region was of curiosity and has remained to be explained (Lee et al. 1998). In the next we check whether a specific magnetic field configuration can explain the RCP in region $B$.

\section{Magnetic Field Models for the QT layers}

We create three magnetic field models for this active region using combinations of six point charges placed at the center locations of S1-S6 (Fig. 1). The first model has all potential field charges with their relative strengths and depths chosen to reproduce the photospheric longitudinal magnetogram as closely as possible. The second and third models are created by adding charges of linear force-free fields only at the locations of S2, S3, S5, and S6 so that we have local electric currents confined in those regions with strengths gauged by the force-free parameters, $\alpha=-2.1 \times 10^{-5} \mathrm{~km}^{-1}$ and $\alpha=-2.9 \times 10^{-5} \mathrm{~km}^{-1}$, respectively. We must note that this is not a standard procedure for constructing the nonlinear force-free field, but should be regarded as a tentative experiment to quickly check how the magnetic field deviates from the potential field configuration in the presence of local currents. In Figure 2 we plot the coronal PILs projected into the sky plane predicted by each model for heights of $h=30^{\prime \prime}, 60^{\prime \prime}, 100^{\prime \prime}, 140^{\prime \prime}$, and $180^{\prime \prime}$, respectively, over the longitudinal magnetogram of the Mees Solar Observatory (MSO). Here the coronal PIL means the points on a coronal plane of height $h$ where the line-of-sight magnetic field vanishes. A set of these coronal PILs at selected heights is shown in Figure 2 as way to visualize the shape of the QT surface. The potential field model shown in the leftmost panel predicts a monotonic shift of the magnetic neutral line to the south as height increases. This explains the depolarization strip in region A, but not the strong $\mathrm{RCP}$ in region $\mathrm{B}$, because emissions from $\mathrm{S} 6$ then pass through the overlying region with the negative magnetic polarity to be observed in LCP. The other two models also predict the general shift of the negative magnetic polarity toward southward, although the predictions differ in the slope of the QT surface with respect to the line of sight. A noticeable difference between the models appears in region B. Here, due to the presence of electric currents, the coronal PIL rotates counterclockwise with height. The degree of 
rotation increases in proportion to the amount of electric current assumed to flow in the photosphere. In particular the third model predicts that the coronal PIL at $h=180^{\prime \prime}$ be oriented almost along the north-south direction above the spots S3 and S6. In this case the radiations in region $\mathrm{B}$ are emitted from the positive magnetic polarity layer and pass through the overlying regions also filled with the positive polarity fields and will be observed as RCP. The third magnetic field model therefore explains the strong RCP in region $\mathrm{B}$ as well as the depolarization in region $\mathrm{A}$.

\section{Discussion}

The high resolution VLA observations of AR 6615 show that radio depolarization strip above a complex active region can be quite sophisticated compared with a simple expectation from the photospheric magnetograms. Although the dominant LCP over the active region could be understood in terms of the southward shift of magnetic PIL with height and mode coupling in the high corona, the strong RCP seen in the western side of this active region was a puzzle as pointed out in the previous study (Lee et al. 1998). A simple modeling presented in this paper oers an alternative interpretation that due to strong currents flowing in the western part of the active region the coronal magnetic PIL rotates with height counterclockwise so that the RCP emission from the region could remain as RCP all the way to the observer. Note that this diagnostic power increases with the number of observing frequencies. With radio polarization maps available at multifrequencies, we can even trace the change of PIL orientation with height, as the depolarization strip appears close to the photospheric PIL at higher frequencies (low corona) and moves gradually away from it at lower frequencies (high corona). This procedure will reveal the location of the QT layer as a function of coronal height, which, in turn, depends on the magnetic field and currents underneath. Microwave polarization maps may thus serve as a powerful diagnostic for the large scale field structure in the outermost active region corona where no other radiation is so sensitive to magnetic field.

The presentation of this paper in the IAU Symposium 273 was possible due to partial support from the NSF grants ATM 0548260, AST 0968672 and NASA LWS grant 09LWSTRT09-0039. J.L. has been supported by NSF grant AST-0908344.

\section{References}

Alissandrakis, C. E., Borgioli, F., Chiuderi Drago, F., Hagyard, M., \& Shibasaki, K. 1996, Solar Phys., 167, 167

Alissandrakis, C. E., Chiuderi-Drago, F. 1994, ApJ, 428, 73

Cohen, M. H. 1960, ApJ, 131, 664

Dalgarno, A. \& Layzer, D. 1987, Spectroscopy of astrophysical plasmas, Cambridge U. Press, Cambridge and New York

Dulk, G. A. \& McLean, D. J. 1978, Solar Phys., 57, 279

Kakinuma, T. \& Swarup, G. 1962, ApJ, 136, 975,

Kundu, M. R. \& Alissandrakis, C. E. 1984, Solar Phys., 94, 249

Lee, J., White, S. M., Kundu, M. R., Mikic, Z., \& McClymont, A. N. 1998, Solar Phys., 180, 193

Lin, H., Penn, M. J., \& Tomczyk, S. 2000, ApJ, 541, L83

Ratcliffe, J. A. 1959, The Magneto-ionic Theory and its Applications to the Ionosphere, Cambridge U. Press, Cambridge

Ryabov, B. I., Pilyeva, N. A., Alissandrakis, C. E., Shibasaki, K., Bogod, V. M., Garaimov, V. I., \& Gelfreikh, G. B. 1999, Solar Phys., 185, 157

Zirin, H. 1988, Astrophysics of the Sun, Cambridge U. Press, Cambridge and New York 